THE $11^{\text {TH }}$ EDITION OF

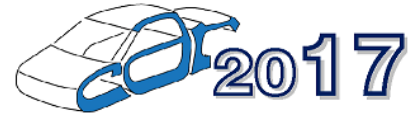

\title{
Solution for eliminating defects caused by improper operation starting execution
}

\author{
Ancuta BALTEANU \\ no. 1, 110040, Pitesti, Arges, Romania \\ *Corresponding author e-mail: a_balteanu@yahoo.com \\ Article history \\ Received 10.06.2017 \\ Accepted 23.09.2017 \\ DOI https://doi.org/10.26825/bup.ar.2017.001
}

University of Pitesti, Faculty of Mechanical and Technology, Targul din Vale str,

\begin{abstract}
Engineering industry represents everything that is developing, manufacturing and selling cars, being one of the most important economic sectors in the world to bring income. In general, companies in this sector are multinationals that produce in their country of origin or to countries that have purchased or built production plants. The main objective of this paper was to study on improving the operation of the post of starting diesel engine. Failure to comply with the priming operation adversely affects the quality indicators on the plant. All these problems have forced to find solutions for their elimination. In order to eliminate the problems, a thorough analysis of the post has been started. Difficulties in finding the solution consisted primarily in the lack of funds to purchase a new priming plant, as the current installation is an integral part of the tank filling facility. This study aims to find solutions to eliminate failures due to improper execution of this operation, defects which are registered in the program that handles the top factory defects and which is calculated based on one of the most important indicators of quality.
\end{abstract}

\section{Introduction}

The company in which this study was conducted is structured on Departments, including the Department of Montage. In this department, the car is equipped with all components, following the production schedule according to the demand on the respective market, from the painted body stage to the finished product stage.

The car body is shifted automatically, on the ground and with the help of air conveyors - in the Assembly Department - from one post to another, in order to assemble the sub-assemblies.body. 
The production system is tactfully set and operates 24 hours a day, with less meal breaks and technological breaks [1]. At the exit of the mounting strip, the cars are filled with fuel so they can be moved to the test stands, where they will go to the Sales Department.

The fuel filling operation is carried out in the Montage Department with a semi-automatic plant, made by a filling company, in the fuel filling station.

For diesel engines, everything in this post is made and operation of fuel priming circuit. The operation of the circuit priming fuel from fuel tank means bringing the high-pressure pump, in order to ease starts [2].

Failure to comply with the Priming operation adversely affects the plant quality indicators by: recording the failures managed by the filling system, recording malfunctions in the vehicle computer due to difficult starting, damage to some product components (starter, battery), increasing the number of operations without Value, ergonomic penalization for manual priming, interruption of production flow through product storage in the retouch area.

\section{Analysis of the priming operation of the system and the starting system of the diesel type fuel circuit}

The primer system uses the vacuum technique. This technique is mainly based on the aspiration phenomenon and uses the phenomenon of creating a depression by using a vacuum generator called an ejector.

The vacuum generator consists of a constriction, followed by a widening of the air passage (divergent). Between this vacuum generator and the fuel, a cylinder is dropped to drop the fuel in it. The starter cycle can only begin when 3 liters of diesel is introduced into the tank. This information is transmitted to the machine by a volumetric counter which generates electromagnetic pulses by rotating a turbine driven by the passage of fuel through its interior [3].

When the number of pulses represents 3 liters of diesel - the minimum threshold set - the starting cycle is automatically launched, of course, if the operator has previously coupled the torch to the priming valve - provided on the turbine-gas line immediately next to the pump high pressure figure 1.

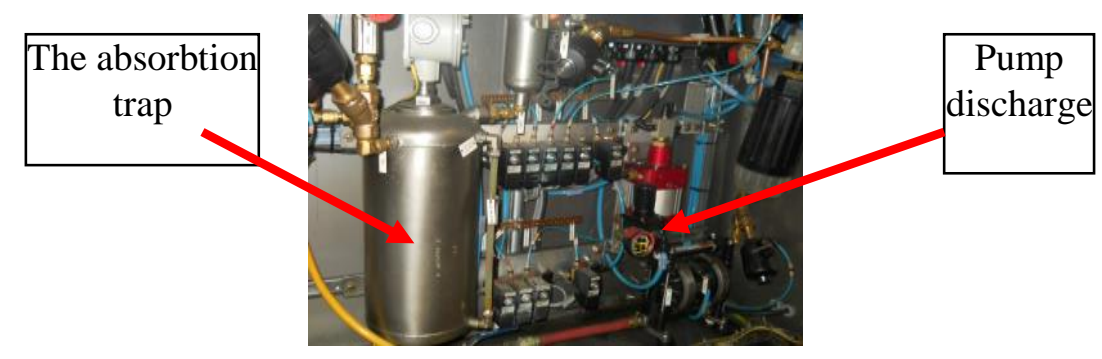

Figure 1. The installation of priming

Diesel fuel is absorbed with the vacuum generator, which can absorb up to 100 mbar absolute pressure. This depression is accomplished with the help of a bottle called the absorption trap.

This device has been given this name because inside it is decanted the diesel that has been absorbed from the tank and, implicitly, from the fuel circuit.

Opening the circuit between the trap and the priming gun is done by means of a pneumatically controlled valve.

The seal of the torch on the car's valve is made by means of an "O" gasket inside the gun. After completing the 30 seconds of absorption, the starter pistol - which is equipped with a pneumatic piston inside it - closes the starter valve on the passenger car.

The maximum capacity of the absorption bottle is about 10 liters. The fill level of this absorption bottle is controlled by some level detectors located inside it. 
The time set from the programmable startup operation is 30 seconds. When the absorption trap has already accumulated 4-5 liters of diesel, it is necessary to empty it in the tank of the car, using a Husky-type pneumatic pump and through the fuel filler pistol.

The flow rate of this pump is 7 liters / min. In the case of a new car, the successful start of the car in optimum conditions means that the fuel line from the tank and the injection pump is full of fuel. In this respect, a manual priming pump is mounted on the circuit between the tank and the filter figure 2 .

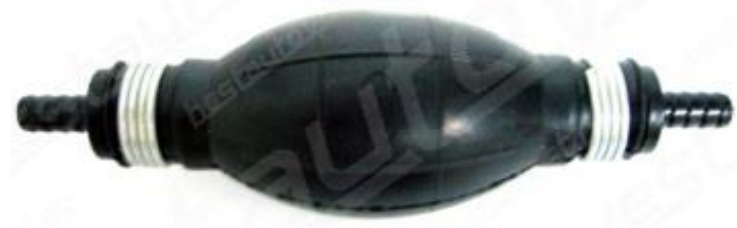

Figure 2. Manual Pump starting

The operating principle of the manual priming pump is that it is made of oil resistant material but also elastic at the same time to allow easy manual pressing [4].

Inside the manual starter pump - at the ends - there are two sense valves, positioned in the same sense as operating principle, an absorption valve and a discharge valve.

Thus, by pushing only one valve, the fuel exits, and by releasing the other valve, the fuel enters the pump. The manual starter pump also has 2 connectors at the ends for mounting on the fuel supply pipe of the car.

By pressing manually, the fuel - located in the inner cavity of the pump since the previous push - is pushed into the diesel fuel filter on the appropriate valve allowing the fuel to flow in that direction.

The loss of hand contact causes the manual pump to be pulled out and, with this, allows absorption of the fuel through the absorption valve, which is opposite the discharge valve, thus the fuel enters the inner cavity of the pump.

The rhythmic repetition of this procedure will lead to the filling of the diesel fuel filter and, implicitly, to the filling of the high pressure pump absorption circuit.

This operation is generally intended and recommended for the case of a vehicle already in use, or when replacing fuel system elements - such as diesel fuel, injectors, pipes, pumps, etc.

In the case of automotive manufacturers, the fuel pipe suppliers have a valve on the high-pressure pump connection line to facilitate the primary fueling operation.

With this valve, the fuel circuit is primed with advanced technical means, which reduces the fueling time of the fuel circuit and improves the quality of the starting operation.

\section{Analysis of causes of defects priming fuel circuit}

\subsection{Identifying the effects of the non-conforming starter operation}

One of the major monitoring indicators of factory production results is STR - Straight Through Ratio. This STR quality indicator is the number of machines that do not come aut with defects from the workflow. The data provided with this indicator is analyzed at management meetings.

On the basis of these values, the necessary measures to improve the situation are discussed, as well as the elaboration of action plans for the reduction of the most damaging defects.

Thus, it was found that in the 'top of the 10 defects' on the plant - revealed by STR - the first two places are the defects called "Nonconforming Priming" with 828 appearances and "Specific Incorrect Operation" with 558 appearances.

Also, the defects obtained on the fuel filling installations have been analyzed in detail - Figure 3. 


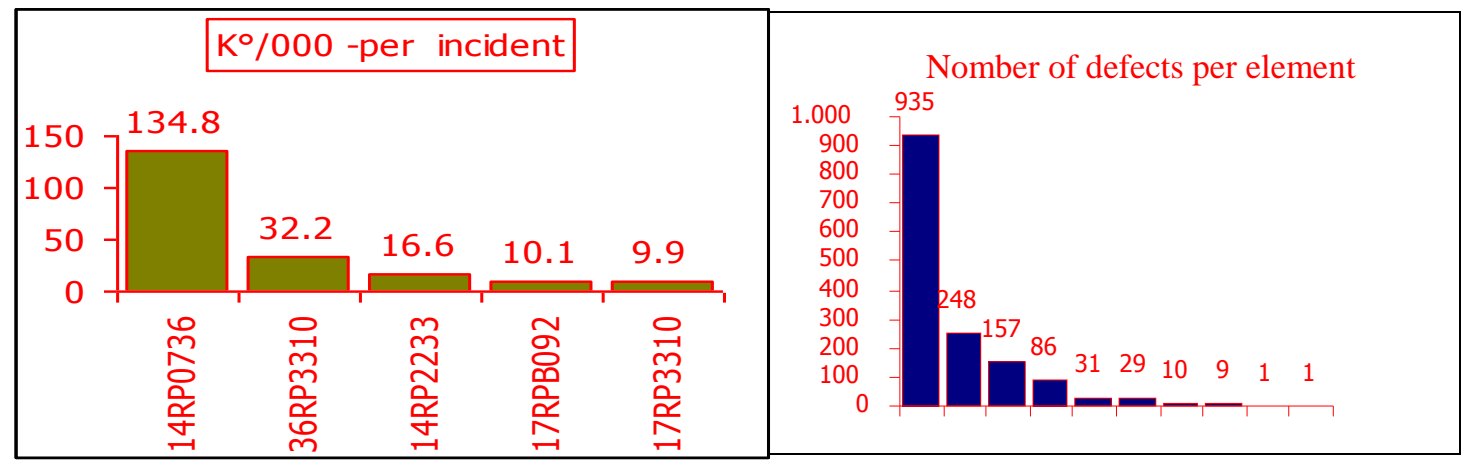

Figure 3. Faults on fuel filling installations

Thus, carrying out the priming operation led to the record of the fault in the fuel filler system, namely "Specific operation not performed".

This defect is recorded by the filling machine if any of the filling operations performed in this post are not performed - for example fuel filling, windshield washer filling, priming. In this situation, it is not known exactly which of these operations are not performed.

Thus, failing to initiate or partial priming can result in the defects recorded in the vehicle's computer, documented under "injection". This defect is recorded due to the heavy starting and the high starting time of the starter.

Deleting the defect recorded in the car computer involves connecting a console with a special software to the car computer.

But this operation is not part of the production process and is not available to any operator. Thus, this correction operation becomes a non-valuable operation and leads to the growth of another indicator called DSTR, which is additional labor / car expenses.

To reduce incidents of this kind, it was necessary to bring an operator who has the task of making the manual pump priming.

This new operation has an average of 180 seconds and determines wrist fatigue while penalizing ergonomic surgery.

Another effect of the first non-compliant start of the car is the premature wear of the starter and lowering the battery voltage.

All these defects will require additional time and costs to remove them.

\subsection{Primary station analysis}

In order to find the solution for defect defect "Specific operation not performed", drawings were drawn from the fault history of the automaton in order to determine the exact type of operation that is not performed according to the procedure.

Thus, as a result of the analysis of the history, it was concluded that the defect "Specific operation not performed" actually belongs to the priming system, and the fuel filling operation was performed because there is the amount loaded in the corresponding box.

Also, starting from the defect information and on what type of cycle, it appears from the analysis tables that it appears on the diesel load cycle.

An analysis of the components of the primer system was then carried out and the functioning and condition of each component analyzed.

Thus, in order for the absorption of diesel fuel - in principle the priming of the circuit - to be carried out at the operating parameters, the circuit first requires leakage.

For this purpose, actions have been taken to verify:

- jector feed pressure,

- if the ejector feed manifold opening is made at time $t$ in the cycle,

- if the opening between the intake trap and the priming torch is made at 3 liters of fuel,

- tightness of the starter gun on the car (ring "O"), 
- closing the valve on the car after the end of the cycle,

- absorption trap has no fuel inside.

From the analysis of the actions carried out, no conclusions were drawn regarding the malfunctioning of the starting system regarding the functioning mode.

Another cause of the priming operation nonconformity occurs when there is fuel in the absorption trap, and the machine receives a signal from the level detector to discharge it.

Discharging the fuel from the absorption trap is done in the tank of the car and takes 25 seconds (43 cmin).

Only after this unloading has been received confirmation that the priming operation can start, thus exceeding the production cycle - the cadence - which is $92 \mathrm{cmin}$.

Another cause of the non-compliance of the priming operation that leads to the failure of the Fuel Filling Plant to operate in the production cycle is the records of the plant history of the defects of the following types: "Mobile Station unavailable".

This defect occurs when the car enters the identification area and the mobile station is not originally due to the fact that the diesel fuel filling operation has been prolonged with the starting operation - Figure 4.

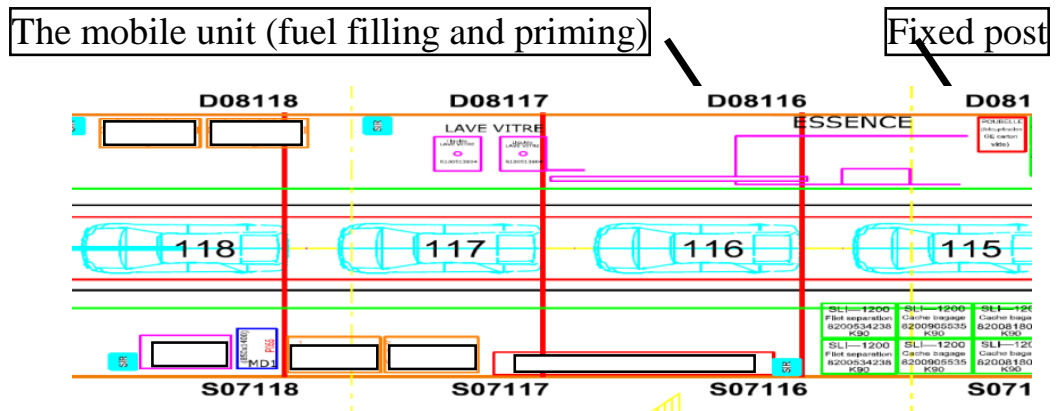

Figure 4. Manufacturing flow in the fuel filling post

It is therefore concluded that the main factor due to which the Diesel Motor Fuel Filling System does not fit into the manufacturing cycle is the increase in the mounting bandwidth from 61 auto $/ \mathrm{h}$ (98cmin.) to 65auto / h $(92 \mathrm{cmin})$.

\section{Solutions to improve the Diesel fuel circuit initiation operation}

Based on the analyzes made in the previous chapters, two directions are required to improve the priming operation.

These directions are: Primary Defects Reduction Actions and Primary Defects Removal Actions.

Due to the fact that the actions for the elimination of defects consist mainly in the moving of the starting station and the introduction of another priming plant - both time and additional costs - in the first phase actions were taken to reduce the existing defects [5].

\subsection{Actions to reduce the existing defects of priming}

In order to reduce the number of defects that occurred during the start-up operation, a series of short-term actions aimed at achieving this objective were planned, namely:

- reducing the amount of diesel from which the starting operation starts,

- reducing the amount of diesel discharged from the absorption trap into the tank,

- improving the vacuum level on the absorption line,

- shortening the time for the priming operation.

All defect reduction actions involved intervention in the automation program that manages the fuel filling cycles to modify some parameters. Thus, the following changes were applied and the following results were obtained: 
- reducing the amount of diesel from which the starting operation starts: Change the fill quantity in the tank to start the starting cycle from 3 liters to 2.5 liters. This change was made from the machine's automaton via the human-machine interface.

- reducing the amount of diesel discharged from the absorption trap into the tank: it intervened in the program so that when the level detector in the absorption trap triggers its emptying into the tank, it will empty one liter of fuel. To avoid the repetitiveness of the latter (trapping) - which would lead to a repeat offset of the fill cycle - the operator trained to manually trap the trap in each technological break by the operator supervising the plant process.

- improving the vacuum level on the absorption line: this parameter was achieved by opening the vacuum circuit between the vacuum generator and the absorption trap at the start of the filling operation instead of starting the priming operation.

- decreasing the time for the priming operation: the starting time cycle has been reduced from 30 seconds to 25 seconds. The action consisted in reducing total cycle time and was due to improved vacuum.

\subsection{Actions to eliminate the existing defects}

Actions of the second phase, aiming at a long-term time horizon, was taken into account:

- the conclusions drawn from the study of the fuel circuit on the Diesel engine, namely the time taken for the fuel to enter the vessel's lithometer probe.

- the fact that it is necessary to detach the priming operation from the fuel filling station and the positioning of a new downstream post at a distance of at least one cycle step from the filling station. As mentioned above, a cycle step is equal to $92 \mathrm{cmin}$.

- the difficulty of short-term acquisition of a new start-up facility, which involves a new investment, for the purchase of such a facility by specialized suppliers. The price required by suppliers for a prime plant is about $€ 50,000$. Due to the high price of a new starter installation, it was proposed to choose the solution to internally build this plant, based on its own design [3].

In the design of the new primary priming plant, the following requirements were met:

- improving the vacuum level with the possibility of adjusting it,

- obtaining components and automating the plant using pneumatic elements,

- realization during priming and the diesel fuel injection function in the tank,

- the possibility of moving the starter gun with the car,

- the facility to implement the new plant in the production stream where, due to automation, much of the starting cycle is masked,

- investments in the installation elements up to the $2000 €$ threshold.

Therefore, the actions taken in the second direction to improve the priming operation and taking into account the above-mentioned requirements together with the results obtained were the following:

- improvement of the vacuum level with the possibility of adjusting it: In the newly made priming system, the vacuum generator (ejector) was replaced by a vacuum pump on which an atmospheric line was mounted in order to regulate the absorption level. This has led to a 100 -fold increase in vacuum performance.

- obtaining components and automating the plant using pneumatic elements: the choice of pneumatic equipment was based on the elimination of the fire risks, but also on their increased reliability.

- making during tank priming and diesel fuel injection function: in order to speed up the filling of the tank in the tank and also to eliminate the risk of the vessel remaining empty during absorption, a diesel fuel injection circuit was implemented in the installation starts at the time $(t+3)$ from the start of the priming operation. This operation is a masked operation in the sense that it is done during fuel absorption and solves the problem of fuel accumulation 
in the absorption trap, so the amount of diesel fuel absorbed from a car is injected into the tank of the next car.

- the possibility of moving the priming torch with the car: this action involved the creation of a mobile station consisting of a frame placed between the new starter and the car on the belt. This mobile station travels by means of trolleys on a suspended rail.

- facilitating the implementation of the new plant in the production stream: the new installation was of limited size, did not require a special spatial arrangement. The new post was intercalated before the second post - downstream from the fuel filling station - Figure 5.

- investment in plant components up to the $2000 €$ threshold: most of the components of the facility were part of the disinfected equipment. Thus, the vacuum pump required only a repair and a repair kit worth $800 €$ was purchased and the prize torch was purchased at the value of $400 €$.

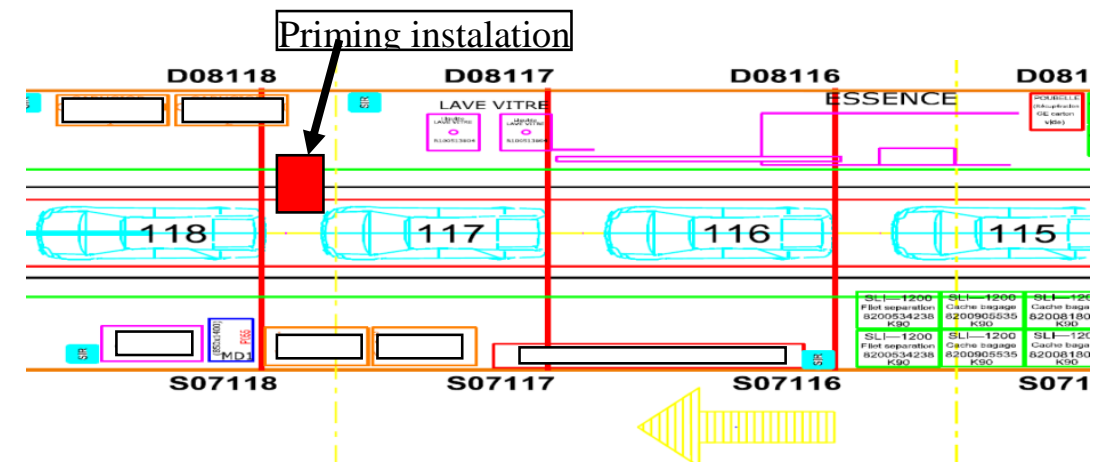

Figure 5. Implementation of priming station

\section{Conclusions and results}

The starting point of the analyzes was the determination - on a fuel circuit equipped with a segmented fuel tank - of the time, amount of diesel and the vacuum level required to carry out a proper priming operation [6].

As a result of these analyzes, it was concluded that due to the increase in the manufacturing cadence of the product and the launch of several Diesel engine models - launching due to market demand for the respective products, sometimes even consecutive series of 100-200 of these cars once - the existing priming plant no longer fits into the production cycle, and the priming operation at the end of the filling cycle was done partially or not at all.

From the analysis carried out, it appeared that at least 1 post $(1 \mathrm{car})$ distance was required between the filling of the tank and the priming operation.

In order to meet this requirement, it was proposed to operate in two directions: the first direction would be for action of diminishing a priming defects, and the second direction would take action to eliminate priming defects.

A solution covering both directions of action is to design and introduce a second installation of priming the fuel circuit, to be located downstream of the tank filling station.

Also, the costs of acquiring a starting facility were analyzed. Thus, in the market for suppliers of such installations, the cost for such an installation is between $50-60 \mathrm{~K} €$. In this situation, it has been proposed to design and execute a primer system that meets the normal requirements and requirements of the field.

The final solution resulted in moving the starter station to at least 1 cycle step ( 1 car) than tank fill operation and position identification.

Finally, the new design and implementation of the fueling system will also bring substantial investment for the company - on the one hand, and on the other hand the elimination of the priming 
problems in the production stream will be a good practice applicable to the other Factories of the company [7].

The main result obtained in improving the priming operation consisted in the implementation of the equipment in the production process. Following this implementation, the following results were achieved:

- after the introduction of the new post, the first start was reduced from $50 \mathrm{cmin}$ to $30 \mathrm{cmin}$ Figures 6, 7.

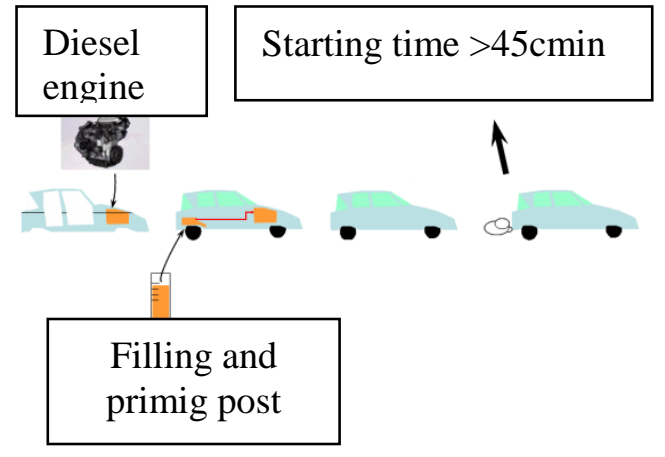

Figure 6. Priming operation same time as filling

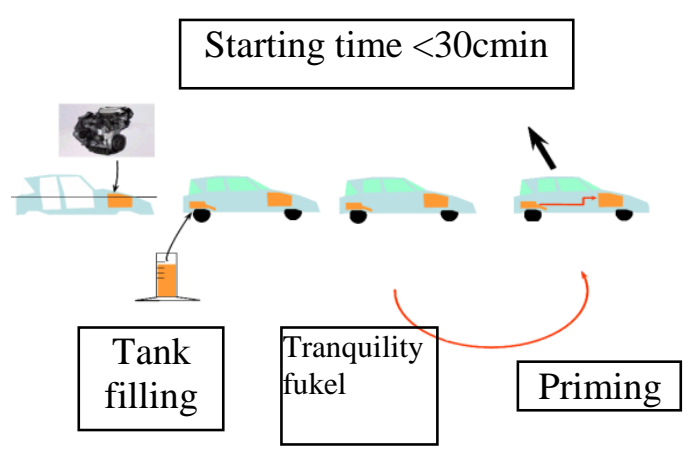

Figure 7. Primig operation offset by filling

- the elimination of the starting defects led to a decrease in the number of cars sent to retouch.

- by eliminating the defects, the quality indicator on the plant - the STR has improved.

- removal of manual start operation, ergonomically penalized.

\section{References}

[1] Clegg B and MacBryde J 2013 Trends in modern operations management, International Journal of Operations \& Production Management, ISSN: 0144-3577 pp. 201-216

[2] Rajenthirakumar D and Harikarthik S G 2011 Lean manufacturing, Implementation in a Construction Equipment Manufacturing Company, Bulletin of Engineering Vol. IV ISSN 2067 - 3809 pp.117-122

[3] Anderson J D Jr 2013 Modern Compressible Flow, with Historical Perspective, 3rd Edition McGrawHill

[4] Fitzpatrick R 2013 Oscillations and Waves: An Introduction. CRC

[5] Kleindorfer P R Singhal K Van Wassenhove K L 2017 Sustainable Operations Management, Production Operations Management Journal, pp. 79-92

[6] Straka L and Krehe R 2014 Determination of the suitability of the method used for evaluation measurement equipment capability, Applied Mechanics and Materials 616:118-25 SCOOPUS

[7] Voss C Tsikriktsis N Frohlich M 2017 Case research in operations management, International Journal of Operations \& Production Management, ISSN: 0144-3577 pp 47-58 